\title{
Medical B-Mode Ultrasound Imaging Reconstruction Algorithms: Evaluation and Simulation
}

\author{
Khadeja M. Mohamed \\ Electronics \& Communications Engineering \\ Al-Nahrain University \\ Baghdad-Iraq \\ Khajjoo.altaay91@gmail.com
}

\author{
Dr. Mohammed H. Ali \\ Electronics \& Communications Engineering \\ Al-Nahrain University \\ Baghdad-Iraq \\ Malhayani@gmail.com
}

\begin{abstract}
Ultrasound is one of the most important imaging modalities in medical practice. It is the most technique development with a lot benefits and with little challenges that include low imaging quality and high variability. Medical field is the most application that exploit the ultrasonic technique widely in body imaging, especially the real time Feature which take advantage of diagnostic time.
\end{abstract}

This research makes a survey on the ultrasound image reconstruction and Display. First, the Ultrasound Imaging are reviewed as overall studying include the frequency ranges, advantages, main limitations, and applications. Second, the 2D or B-mode ultrasound imaging according to generation steps and mathematic studying are reviewed. Third, the stages of the 3D ultrasound object formation which is the data acquisition, data preprocessing, reconstruction method and 3D visualization, are discussed. Fourth, MATLAB Simulation to create B-Mode Ultrasound image with Synthetic phantom of a fetus in the third month of age, by using Field II software made specially for ultrasound environment, after initializing the ultrasound environment a phantom used to yield the BMode image. The complete B-Mode fetus scanned image creation need 5 hours and approximately 20 mins, that each line takes around $2 \mathrm{~min}$ and $30 \mathrm{sec}$, with a processor $1.8 \mathrm{GHz}$ Dual-Core Intel Core i5, MacOS. When the parameters of transducer have been changing then the clearness of the generated image the time taken will change in response.

Keywords: Ultrasound, Echo Imaging, Speckle noise, 3D Reconstruction, Ultrasound Applications, Reconstruction, BMode, Rejoin of Interest (ROI

\section{INTRODUCTION}

Ultrasonography displays the cross-sectional view of the object being scanned. The whole operation is the emitting of sound waves have high frequency into the object, then the reception, processing and display of echoes returning from the organs and tissues of the ROI. This imaging technique has become popular, as it is portable, and produces images with good resolution without the use of ionizing radiation in a cost-effective manner. This is a minimally invasive technique, and is very useful in the diagnosis of obstetrics. It is used to image the internal organs like heart, liver, gallbladder, spleen, kidney and to get the required thing with muscles, tendons, ligaments, joints and soft tissue. Since ultrasonic imaging is real time, it demonstrates the movement of internal organs, tissues, and blood flow and heart valve functions. Ultrasound images are used to identify the presence of cysts, tumors, and fluid filled sacs. They are also used to examine the superficial structures in the body. The operating frequencies for typical applications of the ultrasound waves are showed in Fig.1. High frequency waves cannot penetrate deeply and thus it is used in imaging superficial organs. Low frequency waves penetrate into the body, to image the deeply seated organs [1].

The main limitation of ultrasound imaging system is the speckle noise in the image. Speckle noise is signal dependent noise, that arises due to the interference of transmitted and reflected ultrasound waves in the region of interest. Speckle pattern is a form of multiplicative noise and it depends on the nature of the tissue being imaged, and various imaging parameters.

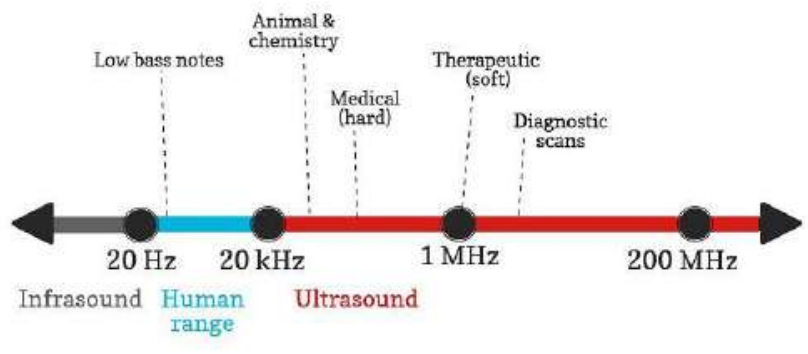

Fig. 1. The operating frequencies for typical applications of the ultrasonic waves

\section{ULTRASOUND APPLICATIONS}

Ultrasound imaging is very useful in many applications because of simplicity and precision imaging for the clinician and patient. The main application of ultrasound imaging which proved its efficiency is in the medical side especially that it is not need to surgery [2].

Ultrasound (US) applications shown in Fig.2. Because of the US ability of disease diagnosis for different of organs in images, fetal development to sports injuries, depending on high or low domain of frequencies, US increased its usage especially among cardiologists and radiologists [3].

Ultrasonic waves including acoustic longitudinal waves which have frequencies more than $20000 \mathrm{~Hz}$ (as shown in fig. 1). US Power is the high-intensity application of ultrasonic energy which is usually higher than $0.1 \mathrm{~W} / \mathrm{cm}^{2}$.

The Power of ultrasonic utilize broadly in ultrasound soldering, welding in plastic and metal, respectively. ultrasonic extraction, ultrasonic cell disruption, ultrasonic trimming, ultrasonic machining, ultrasonic drilling and other high intensity ultrasonic applications where large mechanical displacement magnitude is required, as well as atomization, emulsification and dispersion, degassing and chemical 
reactions in liquids, and metal forming in solids and cleaning [4].

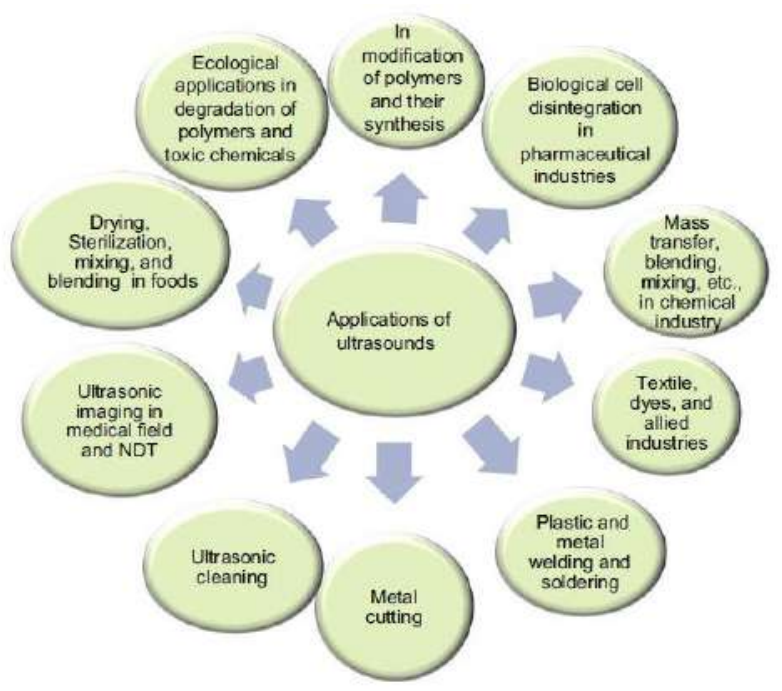

Fig. 2. Some of Ultrasound Applications [17]

\section{A. Ultrasound in Medicine Field}

Ultrasound Imaging has many benefits in comparison to X-Ray, Positron Emission Tomography - Scan Tomography (PET-CT), and Magnetic Resonance Imaging (MRI) [5].

1- Ultrasonic imaging is a kind of nonionizing radiation that made it non harmful in using.

2- US is more simplicity technique than imaging modes of similar uses.

\section{3- US is a real time imaging.}

4- Ultrasound's resolution at millimeter domain for the frequencies, that the resolution be better when the frequency is increased.

5- when applying a Doppler, it can produce blood flow information.

6- It is a small size device.

Although, it has many limitations:

1- There are a finite window has found for US examination of certain organs, such as heart and neonatal brain.

2- US depends on clinician using. At times it is not easy to get acceptable image from patients, including overweight patients [6].

\section{ULTRASOUND IMAGING}

Ultrasound waves work as an information carrier, which are mechanical longitudinal waves of high inaudible frequencies pointed in Fig1. These waves emitted from a probe which acts the sender and the receiver, in time multiplex. This imaging operation based on receiving reflected and scattered waves which are the reaction of the emitted wave (as in radar and sonar systems); this imaging called the echo imaging [4]. When ultrasound wave goes into the body tissue some of these waves will be reflected back and the other keep on travelling through the incoming tissue. These the backing reflected waves create the echo which is picked up by the probe then converted into an electric pulse.
So, the electric pulse goes to the machine to monitor forming image [20].

In practice the Echo imaging much simpler in applying (traditionally called B-scan imaging).

\section{A. Two-Dimensional (2D) Echo Imaging}

Ultrasound Imaging, Echo Imaging, Two-Dimensional Imaging is the same principle of radar or sonar imaging. The transducer gives a short ultrasound wave which propagates in the medium like tissue.

When the waves collide by a particle has impedance differing from the impedance of the medium, it will reflect, refract, or scatter, depending on the size of the object.

The delay $t$ of the received impulse with respect to the emission time determines the radial distance $r$ between the probe and the interface [7].

$$
r=\frac{t}{2 c}
$$

where $c$ is the wave's velocity in the tissue (approximately $1540 \mathrm{~m} / \mathrm{sec}$ ), and the frequency is about 1.5 $\mathrm{MHz}$,

$$
\lambda=\frac{c}{f}
$$

$1540 \mathrm{~m} / \mathrm{sec}=1,500,000(1 / \mathrm{sec}) \lambda$

So, $\lambda=0.001 \mathrm{~m}$

The $0.001 \mathrm{~m}(1.0 \mathrm{~mm})$ is the distance that the wave can propagate in the time available before a new wave starts.

\section{1) Ultrasound B-mode imaging algorithm}

An Ultrasound Image consists of several sequential scanlines (around 128 - 512 scanlines), each of which is usually acquired through transmission and reception beamforming using transducer arrays. The transmission beamforming is to control electronically time delays for ultrasound beam emitted from the transducer arrays to be focused at a point on each scanline. The reception beamforming is to control electronically time delays to accumulate back-scattered echo signals from a specific position, called the received focal point, on scanlines. We note that the received focal point can be changed dynamically on each scanline [8].

The formation of the B-mode images passes through many stages as estimated at Fig.3:

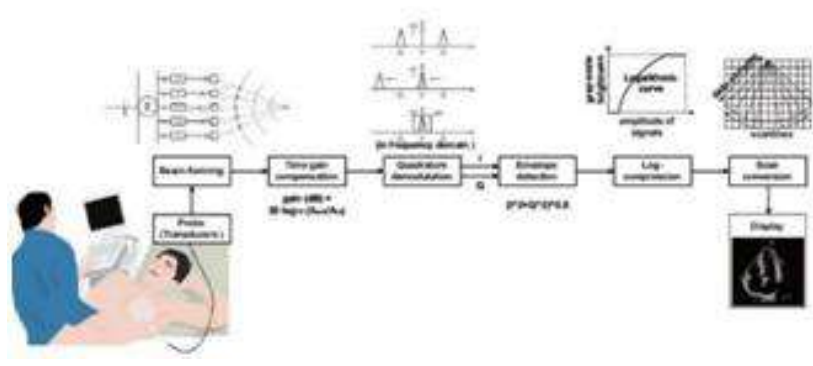

Fig. 3. B-Mode creation stages as mathematics view

1. Beam-forming and gain compensation. Beam forming is a way of form a transmit part that will organize the transmission series from an ultrasound transducer. The transmission pattern is narrow in ultrasound transducer, to 
enlarge it, the transducer will be configured into an array that consists from several crystals or (small transducers) [9].

Let $\mathrm{N}$ be the number of transducers, $\mathrm{r}_{\mathrm{j}}$ the position of the $\mathrm{j}$-th transducer and $\mathrm{p}_{\mathrm{j}}$ the received signal at $\mathrm{r}_{\mathrm{j}}$ for $\mathrm{j}=1, \cdots$, $\mathrm{N}$. By assuming that transducers are very small, sample positions on each scanline are far away from the transducers and the impulse response is given in the form of Dirac delta function. Then, for sample position $r=(x, y, z)$ on a given scanline, the amplitude of the received signal at time $t$ is expressed as

$$
p(t)=\sum_{j=1}^{N}(t-t j)=K \sum_{j=1}^{N} \delta\left(t-\frac{|r j-r|}{c}\right)
$$

where $t_{j}$ is the delayed time and $\mathrm{K}$ a constant depending on depth [18].

2. Quadrature demodulation and envelope detection. Let fo be the center frequency of transmission signals. Then the amplitude of the beam-formed signal along the given scanline can be expressed as

$$
\begin{aligned}
& p(t)=a(t) \cos (2 \pi f \mathrm{o} t+\varphi(t)) \\
& =\frac{a(t)}{2}\left(e^{i(2 \pi \mathrm{fot}+\varphi(t))}+e^{-i(2 \pi \mathrm{fot}+\varphi(t)}\right)
\end{aligned}
$$

where $a(t)$ and $\varphi(t)$ are the amplitude and the phase of the received signal, respectively. In order to remove signals with high-frequency and obtain meaningful signal with lowfrequency, we perform the quadrature demodulation. The baseband complex signal's real and imaginary parts are called In-phase and Quadrature signals, denoted by $I(t)$ and $Q(t)$, respectively.

$$
\begin{gathered}
I(t)=\operatorname{Re}\left(\frac{a(t)}{2} e^{i \varphi(t)}\right) \\
Q(t)=\operatorname{Im}\left(\frac{a(t)}{2} e^{i \varphi(t)}\right)
\end{gathered}
$$

After the envelope detection by taking $\sqrt{ }(I(t) 2+$ $Q(t) 2)$ from the quadrature demodulated signals $I(t)$ and $Q(t)$, logarithmic compression follows.

3. Logarithmic compression. Most of clinically meaningful signals are appeared with the small value in the wide range of amplitude. The logarithmic compression is required to display these meaningful signals in the 256 grayscale values.

The image intensity $I(t)$ is expressed as

$$
\mathrm{I}(\mathrm{t})=\frac{I_{\max }-I_{\min }}{\ln A_{\max }-\ln A_{\min }} \ln \left(\frac{A(t)}{A_{\min }}\right)+I_{\min }
$$

where $I_{\max }$ and $I_{\min }$ are the maximum and minimum intensity values, to be displayed finally in the gray-scale, respectively, and $A_{\max }$ and $A_{\min }$ the maximum and minimum amplitude values with the range of interest, respectively.

4. Digital scan conversion and display. These intensity values at the sample positions along each scanline are displayed on a video screen through scan conversion process for the spatially arrangement. Scan conversion is performed by using bi-linear interpolation.

\section{B. Three-Dimensional (3D) Imaging}

The system of 3D ultrasound imaging displays the ROI in $3 \mathrm{D}$ by forming and combining a group of $2 \mathrm{D}$ frames, that image from multiple positions and angles of ROI [10]. Fig.4 shows the stages:
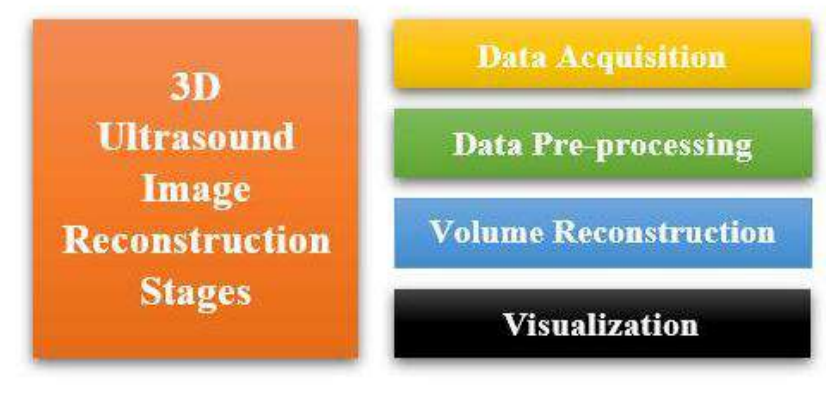

Fig. 4. 3D Ultrasound reconstruction Stages

\section{1) Data Acquisition Stage}

In any way of data acquisition, the main operation is divided into two parts. first, to obtain locations and orientations of the tomographic image's precision, and second, to capture the Region of Interest with speed and efficiency, that aimed to avoid the artifacts caused by cardiac and involuntary motion, also enabling the $3 \mathrm{D}$ visualization of dynamic structures in real time [11]. Fig. 5 shows the types of systems of probe movement.

Linear system uses a side-fire to obtain a cylindrical data volume.

The next three systems are more conventional scanning through the tissue. It is efficient for scanning on small sonic windows, for example the anterior fontanelle or among the ribs.

The next is the pyramidal volume scan that differs with above, the volume is not getting by moving a suitable $2 \mathrm{D}$ probe [10].

Lastly, a freehand system, the movement of probe by hand, it is more suitable way that lets the acquisition of totally arbitrary volumes without the requirement for specialized probe measurements [12].

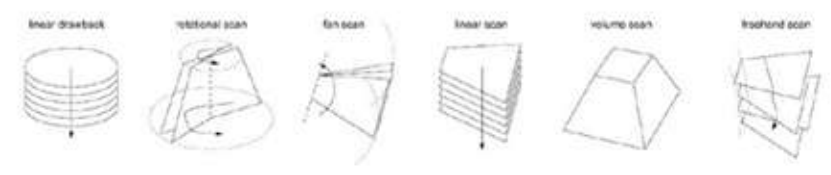

Fig. 5. the Probe's Systems

\section{2) Data Pre-processing}

After the data acquisition, the 2D ultrasonic frames will go to the processing station, to enhance them so most of the processing techniques had been at this stage like Smoothing, Preserve the edge boundary, histogram equalization, 2D Gaussian filter, Median filtering, etc. Ultrasound frames suffer from many noises and artifacts, such as speckle noise, refraction, shadowing, reverberation, etc. [13].

Therefore, a position filtering is necessary to remove high-frequency artifacts. And then enhance the B-scan image by processing technique mention above. The General preprocessing techniques in 3D Ultrasound Imaging are estimated in Fig.6. 


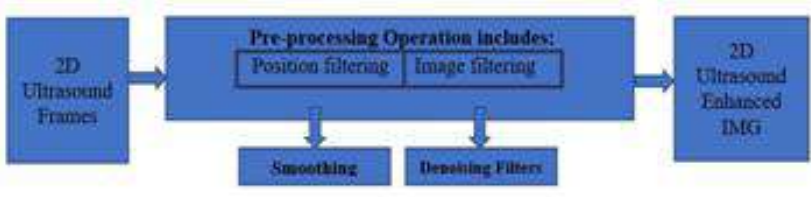

Fig. 6. Steps of the pre-processing stage.

\section{3) $3 D$ Volume Reconstruction}

A mainly part of 3D Ultrasound imaging is $3 \mathrm{D}$ volume construction, and the main step is the determination of organization system configuration for the reconstructed volume (i.e., origin, dimension, and volume grid spacing) [13]. The simplest method is bounding box technique. A bounding box is obtained with the min-point $\left(X_{\min }, Y_{\min }, Z_{\min }\right)$ and max-point $\left(X_{\max }, Y_{\max }, Z_{\max }\right)$. Fig. 7(a) show the origin of the volume is the min-point of the bounding box. The $x$ axis, $y$-axis, and $z$-axis of the volume are obtained with determining the vectors from point 0 to point 1 , point 0 to point 4 , and point 0 to point 3 , respectively. Fig. 7(b) After the volume grid is formed, a following stage is the volume filling stage, it divided into two steps: bin-filling and holefilling [19].

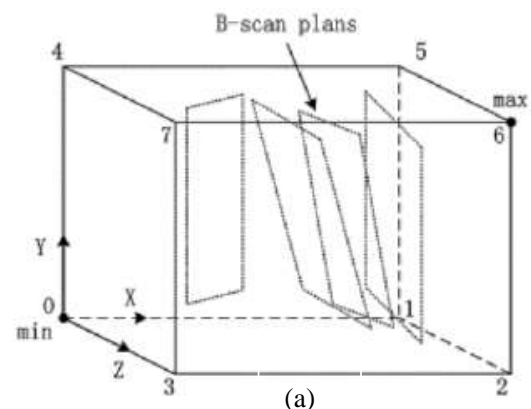

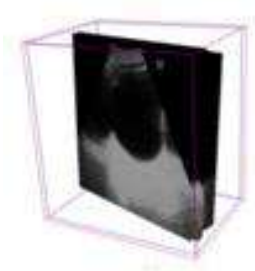

(b)

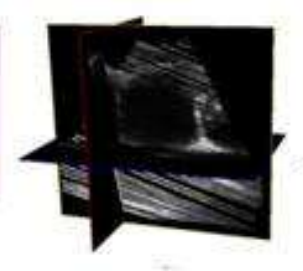

(c)

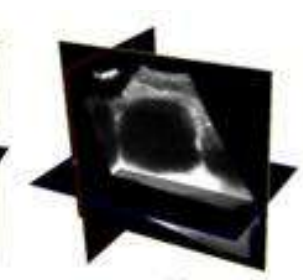

(d)
Fig. 7. 3D Volume reconstruction steps [10] (a) bounding box construction (b) Scanned B-mode images. (c) Hole-filled volume data. (d) reconstructed volume data

Some methods of volume reconstruction as following: pixel-based method (PBM), voxel-based method (VBM), as well function-based method (FBM). Also, the Visualization Toolkit (VTK) is the generality famous software package for volume reconstruction and display [15].

\section{4) $3 D$ visualization/Display}

Next step following volume shaping, the 3D visualization stage is necessary to visualize the volume data from the volume gird for the clinicians to diagnostic the result of probe scanning.

There are three algorithms for 3D visualization are multiplanar reformatting, volume rendering, and surface rendering [24].

Whole operation of $3 \mathrm{D}$ volume reconstruction from the data acquisition to visualization stage in Fig. 8: estimated:
Starting from the top, a 2D sensor is mounted a magnetic position sensing device then the sequence of $2 \mathrm{D}$ frames, pointed with its orientation and position, will combine a coherent data volume [21]. Ending with monitoring images, including planar and non-planar re-slicing and volume rendering.

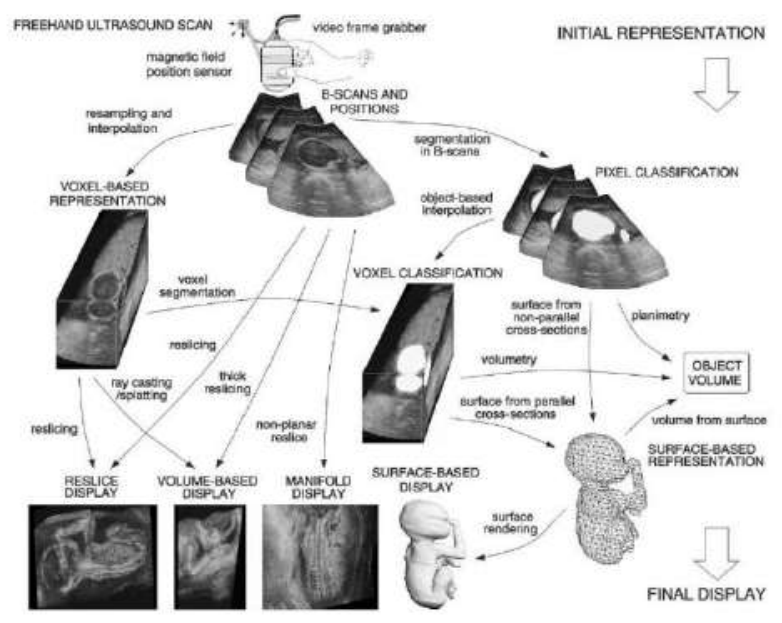

Fig. 8. the main steps of 3D Image reconstruction, from the initial step (top) to the final visualization (bottom).

\section{IMPLEMENTATION AND SIMULATION OF B-MODE IMAGE RECONSTRUCTION}

At this part, the process of B-Mode image creation has presented with parameter's definition and the requirements.

\section{A. The phantom and its Image response}

The phantoms are a way to create images as it shown from real human body. The phantom and ultrasound images are currently $2 \mathrm{D}$, but can readily be developed to 3D image.

A phantom of a 3-month-old fetus (Jensen and Munk 1997), as shown in Fig. 9, this phantom created by 200000 points that distributed randomly these pointes represent scatterers in the tissue.

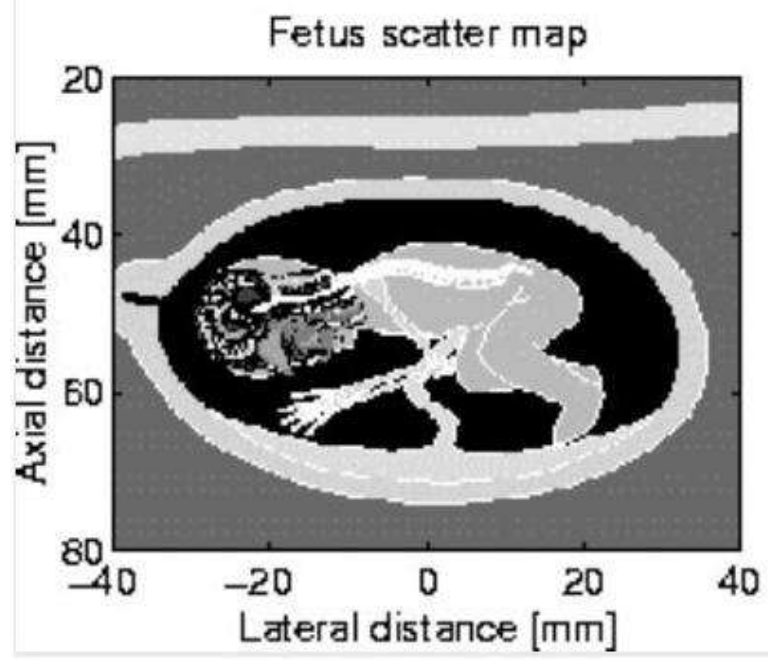

Fig. 9. phantom of 3-month-old fetus [14]

Field II used in [16] and [14], Field II is a program using in simulating ultrasound transducer fields and ultrasound imaging using linear acoustics. The programs use the 
Tupholme-Stepanishen method for calculating pulsed ultrasound fields.

This field depend on linear systems theory to make the ultrasonic environment for both the pulsed and continuous wave state. These make by the spatial impulse response.

The spatial impulse response allows the emitted waves at a certain point in field as a time's function, when the transducer is working by a Dirac delta function.

This program initializes a field system, the phantom is called to make file for the scatterers in it. The simulation of field is represented and the information is saved in RF-files; one for each RF-line done.

This information then should subsequet processed to do the polar to rectangular forming to produce the image. The image created with a dynamic range of $40 \mathrm{~dB}$, the parameters are playing an important role on the execution result.

\section{Algorithm's Parameters:}

Transducer center frequency $=5 \mathrm{MHz}$;

Sampling frequency $=100 \mathrm{Mhz}$;

Speed of sound $=1540 \mathrm{~m} / \mathrm{s}$;

Wavelength: lambda $=0.001 \mathrm{~m}$

Width of element $=$ lambda/2;

Height of element $=7 / 1000 \mathrm{~m}$;

kerf $=0.0025 / 1000 \mathrm{~m}$;

Fixed focal point $=\left[\begin{array}{lll}0 & 0 & 70\end{array}\right] / 1000 \mathrm{~m}$;

Number of physical elements $=64$;

The image consists of 128 lines and value among the lines is 0.7 .

The fetus image resultant exhibited in Fig.10; the image has good features from real scan images. The images are showing large potential to make powerful fully synthetic phantoms, that can be used for image quality evaluation.

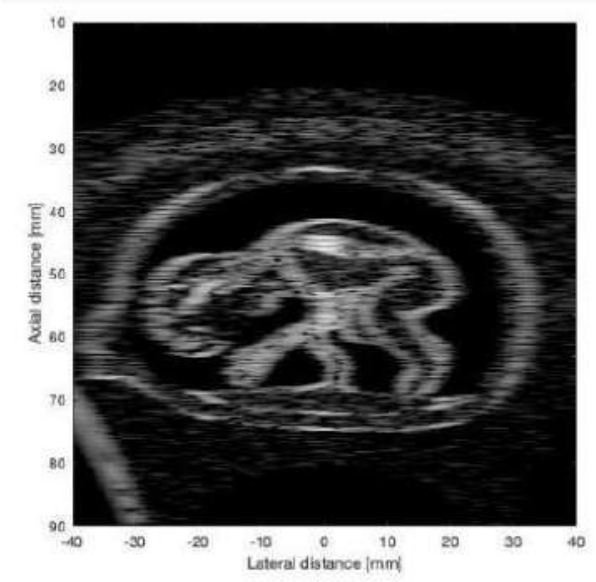

Fig. 10. A Reconstructing B-Mode Image

The changing in the resolution of the result image makes by do modification in the parameters value by try and error method.
When kerf (Spacing between two adjacent elements in transducer) be smaller than its current value that equal to $0.2 \mathrm{~mm}$ the details be clearer but it takes a long time than the current, that make it not suitable to apply, as shown in table 1.

\section{CONClusion}

Because of the non-radiation, easy and non-expensive that made the ultrasound waves suitable for many applications.

The 2D Echo imaging, depended on the probe movement and there are six types of transducer systems, at these days the free hand probe is the most usable.

For the B-mode Image, a fast program for the simulation of ultrasound imaging has been made. It can realistically simulate all kinds of ultrasound systems including color imaging. A full simulation can be completed in 5 hours and approximately $20 \mathrm{mins}$, that each line takes around $2 \mathrm{~min}$ and $30 \mathrm{sec}$, that the image consists of 128 lines with 64 elements of transducer, with a processor $1.8 \mathrm{GHz}$ Dual-Core Intel Core i5, MacOS.

When the parameters of transducer changed then the clearness of the scanned image also had been changing. By trying to make the kerf $=0.2 \mathrm{~mm}$, the running time takes up about 17 hour, that $8 \mathrm{~min}$ for each line, at the same features of machine.

TABLE I. RESULT COMPARISON

\begin{tabular}{|c|c|c|c|c|}
\hline Exp. & $\begin{array}{c}\text { Reconstructed } \\
\text { Image }\end{array}$ & $\begin{array}{c}\text { Trans. } \\
\text { Freq. } \\
\text { (Fo) }\end{array}$ & Kerf & $\begin{array}{c}\text { Taken } \\
\text { time }\end{array}$ \\
\hline$[16]$ & $5 \mathrm{MHz}$ & $\begin{array}{c}2.5 \\
\mathrm{~mm}\end{array}$ & $\begin{array}{c}5 \\
\text { min/line }\end{array}$ \\
\hline$[14]$ & & 7.379 & 0.199 & $\begin{array}{c}25 \\
\mathrm{~mm} \\
\text { min/line }\end{array}$ \\
\hline $\begin{array}{c}\text { Our } \\
\text { Work }\end{array}$ & $\mathrm{MHz}$ & $5 \mathrm{MHz}$ & $\begin{array}{c}0.0025 \\
\mathrm{~mm}\end{array}$ & $\begin{array}{c}2.5 \\
\mathrm{~min} / \mathrm{line}\end{array}$ \\
\hline
\end{tabular}

In the future the resultant B-Mode image can be enhanced by enhancement techniques or be colored image and that the program can develop the $2 \mathrm{D}$ image to $3 \mathrm{D}$ object by display multiple frames.

\section{REFERENCES}

[1] Afonso. M \& Miguel Sanches, "Image Reconstruction Under Multiplicative Speckle Noise Using Total Variation", Neuro computing, Elsevier, 2015.

[2] https://www.vedantu.com/physics/ultrasound

[3] D. C. Levin, V. M. Rao, A. J. Maitino, L. Parker y J. H. «Comparative increases in utilization rates of ultrasound examinations among radiologists, cardiologists, and other physicians from 1993 to 2001, » Journal of the american college of radiology, 2004. 
[4] Ye Yao Yue Pan Shiqing Liu, "Power ultrasound and its applications: A state-of-the-art review" In Ultrasonics Sonochemistry,2019.

[5] Chen Du, Ning-Li Chai, En-Qiang Linghu, Hui-Kai Li, Li-Hua Sun, Lei Jiang, Xiang-Dong Wang, Ping Tang, Jing Yang. "Comparison of endoscopic ultrasound, computed tomography and magnetic resonance imaging in assessment of detailed structures of pancreatic cystic neoplasms" 2017.

[6] Swanirbhar Majumder, Smita Majumder, chapter 6 "Biomedical Image Processing and Analysis", IGI Global, 2017.

[7] Henry Knipe, "https://radiopaedia.org/articles/distance-measurement" 2021

[8] D.R. Dance, S. Christofides, A.D.A. Maidment, I.D. McLean, K.H Ng "Diagnostic Radiology Physics: A Handbook for Teachers and Students", 2014

[9] Sanketh Vedula, Ortal Senouf, Grigoriy Zurakhov, lex Bronstein. "Learning beamforming in ultrasound imaging" In Proceedings of Machine Learning Research, 2018.

[10] Chan Vei Siang, Farhan Mohamed, "A Survey on 3D Ultrasound Reconstruction Techniques", In IntechOpen, 2019.

[11] Qinghua Huang, Zhaozheng Zeng "A Review on Real-Time 3D Ultrasound Imaging Technology”, In BioMed Research International, 2017.

[12] Andrew Gee, Richard Prager, Graham Treece, Laurence Berman "Engineering a freehand 3D ultrasound system" In Pattern Recognition Letters, 2003

[13] D Miller, C Lippert, F Vollmer, O Bozinov, L Benes, D M Schulte, U Sure. "Comparison of different reconstruction algorithms for threedimensional ultrasound imaging in a neurosurgical setting” 2012.

[14] Monika Kofler, Andreas Beham, Michael Affenzeller, Stefan Wagner, "Optimization of medical ultrasound transducers with simulation and genetic algorithms" In Proceedings of the 20th European Modeling and Simulation Symposium, 2008.

[15] Jǐrí Jan, "Medical Image Processing, Reconstruction and Restoration" 2005.

[16] https://field-ii.dk

[17] Lalit Jajpura, Rajkishore Nayak, "Ultrasound applications in textiles and apparels" In Woodhead Publishing Series in Textiles, 2020.

[18] Jaeseong Jang, Chi Young Ahn, "Industrial Mathematics in Ultrasound Imaging" In Korean Society for Industrial and Applied Mathematics ,2016.

[19] K. Kirk Shung. "Diagnostic ultrasound Imaging and Blood Flow Measurements", 2006.

[20] Roelandt, J.R.T.C. "Seeing the invisible: a short history ofcardiac ultrasound", Eur. J. Echocardiography, 2000.

[21] Richard Prager, Andrew Geea, Laurence Berman, Freehand 3D ultrasound without voxels: volume measurement and visualization using the Stradx system, 2003,

[22] Tiexiang Wen, Qingsong Zhu, Wenjian Qin, Ling Li, Fan Yang, Yaoqin Xie, Jia Gu. "An accurate and effective FMM-based approach for freehand 3D ultrasound reconstruction", 2013.

[23] Chan Vei Siang, Farhan Mohamed, Muhammad Ismail Mat Isham, Yusman Azimi Yusoff, Ahmad Ashraf Abd Aziz, Dyah Ekashanti Octorina D. "A Framework of Position Tracked Freehand 3D Ultrasound Reconstruction Using Game Controller and Pixel Nearest Neighbour Method for Marching Cubes Volume Visualization" ,2018.

[24] Honggang Yu, Marios S Pattichis, Carla Agurto \& M Beth Goens. "A 3D Freehand Ultrasound System for Multi-view Reconstructions from Sparse 2D Scanning Planes" In Bio Medical Engineering Online, 2011. 